Pacific Journal of Mathematic 


\title{
NORMALIZERS OF $p$-SUBGROUPS IN FINITE GROUPS
}

\author{
George Glauberman
}

\begin{abstract}
In a recent paper, Sims obtained some striking applications of graph theory to group theory. Using his work, Wong determined every finite primitive permutation group in which the stabilizer of a point has some orbit of length three. The techniques of Sims and Wong can be applied to other situations that occur in investigations of finite groups. In this paper we obtain some applications that we will use in studying weakly closed elements of Sylow 2-subgroups.
\end{abstract}

TheOREM 1. Suppose $P$ is a subgroup of a finite group $G, g \in G$, and $P \cap P^{g}$ is a normal subgroup of prime index $p$ in $P^{g}$. Let $n$ be apositive integer, and let $\widetilde{G}=\left\langle P, P^{g}, \cdots, P^{g^{n}}\right\rangle$. Assume that:

(1) $g$ normalizes no nonidentity normal subgroup of $P$, and

(2) $P \cap Z(\widetilde{G})=1$.

Then $|P|=p^{t}$ for some positive integer $t$ for which $t \leqq 3 n$ and $t \neq 3 n-1$. Moreover, if $n=2, p=2$, and $t=6$, then $P$ contains $a$ nonidentity normal subgroup of $\widetilde{G}$.

Theorem 2. Suppose $H$ is a subgroup of a finite group $G, P$ is a Sylow 2-subgroup of $\mathrm{H}, \mathrm{O}_{2}(H) \neq 1$, and $\mathrm{H} / \mathrm{O}_{2}(H)$ is a dihedral group. Let $S$ be a Sylow 2-subgroup of $G$ that contains $P$, and let $|P|=2^{t}$.

(I) Suppose $y \in S-P, y^{2} \in P C(P)$, and $y$ normalizes $P$. Assume $y$ does not normalize any nonidentity normal subgroup of $H$ contained in $P$. Then $2 \leqq t \leqq 4$, and $H / K(H)$ is isomorphic to $Z_{2} \times Z_{2}, S_{4}$, or $S_{4} \times Z_{2}$.

(II) Assume the hypothesis of (I), and suppose further that $N_{S}\left(O_{2}(H)\right)=P$. If $t=2$ or $t=3$, then $S$ is a dihedral or a semidihedral group. It $t=4$, then $S$ is a group of order 32 generated by elements $x, y$, and $z$ satisfying

$$
x^{8}=y^{2}=z^{2}=[y, z]=1, \quad[x, y]=x^{6} \text {, and }[x, z]=x^{4} .
$$

(III) Suppose $P<S$ and $N_{S}(Q)=P$ for every nonidentity normal subgroup $Q$ of $H$ that is contained in $P$. Then $H$ satisfies the conclusions of (I) and (II).

Throughout the paper, we will assume that $G$ is a finite group. We will generally use the notation of [5] and [9]. In particular, if $G$ denotes a permutation group on a set $\Omega$ and $\alpha \in \Omega, G_{\alpha}$ will denote the stabilizer of $\alpha$ in $G$. Also, $Z_{n}, D_{n}$, and $S_{n}$ will denote the cyclic group of order $n$, the dihedral group of order $n$, and the symmetric group of degree $n$. 
If $H$ is an element or a subgroup of $G$ and if $g \in G$, we let $H^{g}=g^{-1} H g$. Suppose $H$ is a subgroup of $G$. We let $O_{2}(H)$ denote the largest normal 2-subgroup of $H$ and $K(H)$ denote the 2-regular core, or largest normal subgroup of odd order, of $H$.

2. Applications of Sims' method. The proof of Theorem 1 depends on the arguments in Sims' paper [5]. The following result is essentially a restatement of his Proposition 2.6.

Lemma 1. Let $x$ and $g$ be elements of $G$. Put $x_{i}=x^{g^{i}}=g^{-i} x g^{i}$ for $i=0, \pm 1, \cdots$, and define $H_{i}=\left\langle x_{1}, \cdots, x_{i}\right\rangle$ for each $i \geqq 1$. Let $H_{0}=1$. Suppose that $x$ has prime order $p$ and that there exist positive integers $t$ and $n$ such that

(1) $\left\langle H_{t}, g\right\rangle=G$,

(2) $\left|H_{i}: H_{i-1}\right|=p, \quad 1 \leqq i \leqq t$, and

(3) $H_{t}$ contains no nonidentity normal subgroup of $G$ and no nonidentity subgroup of $Z\left(H_{t+n}\right)$.

Then $t \leqq 3 n$ and $t \neq 3 n-1$. Moreover, if $n=2, p=2$, and $t=6$, then $H_{t}$ contains a nonidentity normal subgroup of $H_{8}$.

Proof. Let $s=t+1$. Suppose $1 \leqq i \leqq t . \quad$ By (1) and (2), $G=$ $\left\langle x_{1}, g\right\rangle=\left\langle H_{i}, g\right\rangle$ and $\left|H_{i}\right|=p^{i}$. By (3), $g$ does not normalize $H_{i}$. Now the proof of Lemma 2.7 of [5] remains valid; that lemma states that $H_{i}$ is Abelian whenever $1 \leqq i<(2 s+1) / 3=(2 t+3) / 3$.

Assume that $t>3 n$ or that $t=3 n-1$. Note that in the latter case, $t+n$ is odd. Let $i=(t+n+1) / 2$ if $t+n$ is odd, and $i=$ $(t+n+2) / 2$ if $t+n$ is even. Then $1 \leqq i \leqq t$ and $2 i-1 \geq t+n$. Therefore $H_{t} \cap Z\left(H_{2 i-1}\right)=1$. Since

$$
H_{2 i-1}=<H_{i},\left(H_{i}\right)^{g^{i-1}}>\text { and } x_{i} \in H_{i} \cap\left(H_{i}\right)^{g^{i-1}} \text {, }
$$

$H_{i}$ is not Abelian. By the previous paragraph, $i<(2 t+3) / 3$, which yields a contradiction.

Now suppose $n=2, p=2$, and $t=6$. On pages 85-86 of [5], Sims proves that

$$
\begin{gathered}
{\left[x_{i}, x_{j}\right]=1 \quad \text { for }|i-j| \leq 3} \\
{\left[x_{1}, x_{5}\right]=x_{3}, \quad\left[x_{2}, x_{6}\right]=x_{4}, \quad\left[x_{3}, x_{7}\right]=x_{5},}
\end{gathered}
$$

and

$$
\left[x_{1}, x_{6}\right]=x_{3}^{c} x_{4}^{d}, \quad\left[x_{2}, x_{7}\right]=x_{4}^{c} x_{5}^{d},
$$

for some integers $c, d$. Therefore,

$$
\left[x_{3}, x_{8}\right]=\left[x_{1}, x_{6}\right]^{g^{2}}=x_{5}^{c} x_{6}^{d}, \quad\left[x_{4}, x_{8}\right]=\left[x_{2}, x_{6}\right]^{g^{2}}=x_{6} .
$$


Thus $H_{8}=\left\langle x_{1}, \cdots, x_{8}\right\rangle$ normalizes $\left\langle x_{3}, x_{4}, x_{5}, x_{6}\right\rangle$. This completes the proof of Lemma 1 .

Suppose $G$ is a transitive permutation group on a set $\Omega$. Let $\alpha \in \Omega$, and let $\Lambda$ be an orbit of $G_{\alpha}$ on $\Omega$. By Propositions 4.1 and 4.2 of [5], there exist $\Lambda(\beta)$ and $\Lambda^{\prime}(\beta)$ for each $\beta \in \Omega$ such that:

(1) $\Lambda(\beta)$ and $\Lambda^{\prime}(\beta)$ are orbits of $G_{\beta}$ on $\Omega$ and $\Lambda(\alpha)=\Lambda$,

(2) $\Lambda(\alpha)^{g}=\Lambda\left(\alpha^{g}\right)$ and $\Lambda^{\prime}(\alpha)^{g}=\Lambda^{\prime}\left(\alpha^{g}\right)$ for all $g \in G$, and

(3) $\beta \in \Lambda(\alpha)$ if and only if $\alpha \in \Lambda^{\prime}(\beta)$.

Let $E=\{(\beta, \gamma) \mid \beta \in \Omega, \gamma \in \Lambda(\beta)\}$. Then $(\Omega, E)$ is called the graph of $\Lambda$. It is undirected if $\Lambda(\alpha)=\Lambda^{\prime}(\alpha)$ and is directed if $\Lambda(\alpha) \neq \Lambda^{\prime}(\alpha)$. By Proposition 4.3 of [5], $G$ acts as a group of automorphisms of the graph of $\Lambda$ and is transitive on both the points and the edges.

LEMma 2. Suppose $g \in G$ and $\alpha^{g} \in \Lambda$. Then the graph of $\Lambda$ is connected if and only if $\left\langle G_{\alpha}, g\right\rangle=G$.

Proof. Let $H=\left\langle G_{\alpha}, g\right\rangle$ and $\Gamma=\left\{\alpha^{h} \mid h \in H\right\}$. Then $\Lambda=$ $\left\{\alpha^{g x} \mid x \in G_{\alpha}\right\}$. By the proof of Theorem 7.4 of [7], $\Gamma=\Omega$ if and only if $H=G$. For every $\alpha^{h} \in \Gamma$,

$$
\Lambda\left(\alpha^{h}\right)=\Lambda(\alpha)^{h}=\left\{\alpha^{g x h} \mid x \in G_{\alpha}\right\} \subseteq \Gamma .
$$

Similarly,

$$
\Lambda^{\prime}\left(\alpha^{h}\right)=\Lambda^{\prime}(\alpha)^{h}=\left\{\alpha^{g^{-1} x_{h}} \mid x \in G_{\alpha}\right\} \subseteq \Gamma .
$$

Thus, if the graph of $\Lambda$ is connected, then $\Gamma=\Omega$.

Conversely, assume that $H=G$. Let $\Phi$ be the connected component of $\alpha$ in $\Omega$. Since $G$ acts as a group of automorphisms on the graph of $\Lambda$, each element of $G_{\alpha}$ maps $\Phi$ onto itself. Similarly, $\Phi^{g}=\Phi$ because $\alpha^{g} \in \Phi^{g} \cap \Lambda(\alpha) \subseteq \Phi^{g} \cap \Phi$. Thus, $\Phi=\Phi^{H}=\Phi^{G}=\Omega$.

This completes the proof of Lemma 2 .

Let us assume the notation of Lemma 2. Suppose $t \geqq 1$. Define a $t$-arc to be an ordered $(t+1)$-tuple of points of $\Omega$, say, $X=$ $\left(\alpha_{0}, \alpha_{1}, \cdots, \alpha_{t}\right)$, such that $\alpha_{i+1} \in \Lambda\left(\alpha_{i}\right), 0 \leqq i<t$. Any $t$-arc of the form $\left(\alpha_{1}, \cdots, \alpha_{t}, \gamma\right)$ is called a successor of $X$, and any $t$-arc of the form $\left(\gamma, \alpha_{0}, \cdots, \alpha_{t-1}\right)$ is called a predecessor of $X$.

LEMma 3. Assume the hypothesis of Lemma 2. Suppose $t \geqq 1$ and the graph of $\Lambda$ is connected. Let $X$ and $Y$ be t-arcs. Then there exists a sequence $X=X_{0}, \cdots, X_{r}=Y$ of $t$-arcs such that $X_{i}$ is a successor of $X_{i-1}, 1 \leqq i \leqq r$.

Remark. For $t=1$, this is equivalent to Proposition 3.1 of [5].

Proof. Let $X=\left(\alpha_{0}, \alpha_{1}, \cdots, \alpha_{t}\right)$ and $Y=\left(\beta_{0}, \beta_{1}, \cdots, \beta_{t}\right)$. By 
Proposition 3.1 of [5], there exists a finite sequence $\alpha_{t}=\gamma_{0}, \gamma_{1}, \cdots, \gamma_{s}=\beta_{0}$ such that $\gamma_{i} \in \Lambda\left(\gamma_{i-1}\right)$ for $i=1,2, \cdots, s$. Let $r=s+t$, and let $\gamma_{i}=\beta_{i-s}$ for $i=s+1, s+2, \cdots, s+t$. Define $X_{0}=X$,

$$
X_{i}=\left(\alpha_{i}, \alpha_{i+1}, \cdots, \alpha_{t}, \gamma_{1}, \cdots, \gamma_{i}\right) \quad(1 \leqq i \leqq t-1),
$$

and

$$
X_{t+i}=\left(\gamma_{i}, \gamma_{i+1}, \cdots, \gamma_{i+t}\right) \quad(0 \leqq i \leqq s) .
$$

Then the sequence $X_{0}, X_{1}, \cdots, X_{r}$ satisfies the conclusion of the lemma.

Proof of Theorem 1. Clearly, we may assume that $G=\langle P, g\rangle$. By condition (1) of the theorem, $G$ is faithfully represented as a permutation group on the set of all $\operatorname{cosets} P x, x \in G$, and $P$ is the stabilizer in $G$ of the point $\alpha=P 1$. Let $h=g^{-1}$ and $\beta=\alpha^{h}$. Then

$$
G_{\alpha \beta}=G_{\alpha} \cap G_{\beta}=G_{\alpha} \cap\left(G_{\alpha}\right)^{g^{-1}}=P \cap P^{g^{-1}} \text {. }
$$

Since $P \cap P^{g}$ is normal and of index $p$ in $P^{g}, G_{\alpha \beta}$ is normal and of index $p$ in $P$. Let $\Lambda$ be the orbit of $P$ on $\Omega$ that contains $\beta$. Then $|\Lambda|=\left|\beta^{P}\right|=\left|P: P_{\beta}\right|=\left|G_{\alpha}: G_{\alpha \beta}\right|=p$. Since $P_{\beta}$ is normal in $P$ and $P$ is transitive on $\Lambda, P_{\beta}$ fixes every point of $\Lambda$. Thus $P$ induces a regular group of permutations on $\Lambda$. We define $\Lambda(\gamma), \gamma \in \Omega$, as above.

Suppose $t \geqq 1$. We define $t$-arcs as above. The arguments of $\S 5$ of [5] now give us the following results:

For some $t_{0}, G$ is transitive on the set of all $t_{0}$-arcs but not on the set of all $\left(t_{0}+1\right)$-arcs (Lemma 5.7).

$G$ acts regularly on the set of all $t_{0}$-arcs, and $|P|=\left|G_{\alpha}\right|=p^{t_{0}}$ (Lemma 5.12; see Lemma 3 above).

For any $t_{0}$-arc $X=\left(\alpha_{0}, \alpha_{1}, \cdots, \alpha_{t_{0}}\right)$, the stabilizer of $\alpha_{0}, \alpha_{1}, \cdots$, and $\alpha_{t_{0-1}-1}$ is generated by a single element $x$ of order $p$. If $X^{k}$ is a predecessor of $X$, then $\left\langle x^{k}, \cdots, x^{k^{i}}\right\rangle$ has order $p^{i}$ for $1 \leqq i \leqq t_{0}$. Moreover,

$$
<x^{k}, \cdots, x^{k^{t_{0}}}>=P \quad \text { (Lemma 5.13). }
$$

Let $t=t_{0}$ and let $X$ be the $(t+1)$-tuple given by $X=\left(\alpha, \alpha^{h}, \cdots, \alpha^{h^{t}}\right)$. Since $\alpha^{h}=\beta \in \Lambda$, we have $\alpha^{h^{i+1}}=\beta^{h^{i}} \in \Lambda(\alpha)^{h}{ }_{i}=\Lambda\left(\alpha^{h^{i}}\right)$ for all $i$. Hence $X$ is a $t$-arc and $X^{h^{-1}}$ is a predecessor of $X$. Let $k=g=h^{-1}$, and take $x$ as in the last mentioned result above. Put $x_{i}=x^{g^{i}}$ for all $i \geqq 1$. Then

$$
\left\langle x_{1}, \cdots x_{t}\right\rangle=P \text { and }\left\langle x_{1}, x_{2}, \cdots, x_{t+n}\right\rangle=\left\langle P, P^{g}, \cdots, P^{g^{n}}\right\rangle=\widetilde{G} .
$$

Now we may apply Lemma 1 to prove Theorem 1 .

CoRollaRY 1. Suppose $P$ and $H$ are subgroups of $G, y \in N(P)$, 
and $a \in H$. Assume that $H=\left\langle P, P^{a}\right\rangle$ and that $P \cap P^{a}$ is a normal subgroup of prime index $p$ in $P$. Let $n$ be a positive integer, and let $\widetilde{G}=\left\langle H, H^{y}, \cdots, H^{y^{n-1}}\right\rangle$. Assume that:

(1) y normalizes no nonidentity normal subgroup of $H$ contained in $P$, and

(2) $P \cap Z(\widetilde{G})=1$.

Then $|P|=p^{t}$ for some positive integer $t$ for which $t \leqq 3 n$ and $t \neq 3 n-1$. Moreover, if $n=2, p=2$, and $t=6$, then $P$ contains a nonidentity normal subgroup of $\widetilde{G}$.

Proof. We merely verify the hypothesis of Theorem 1 for $g=y a$. Now, $P \cap P^{g}=P \cap P^{a}$ and $\left\langle P, P^{g}\right\rangle=\left\langle P, P^{a}\right\rangle=H$. Let $H_{i}=$ $<H, H^{g}, \cdots, H^{g^{i}}>$ for all $i \geqq 0$. Since

$$
H_{i+1}=\left\langle H_{i},\left(H_{i}\right)^{g}\right\rangle=\left\langle H_{i},\left(H_{i}\right)^{y a}\right\rangle=\left\langle H_{i}, H_{i}^{y}\right\rangle,
$$

we obtain $H_{i}=\left\langle H, H^{y}, \cdots, H^{y^{i}}\right\rangle$ by induction. Therefore,

$$
\left.\widetilde{G}=H_{n-1}=<H, H^{g}, \cdots, H^{g^{n-1}}\right\rangle=\left\langle P, P^{g}, \cdots, P^{g^{n}}\right\rangle .
$$

Now Theorem 1 applies.

3. Applications of Wong's method. To obtain Theorem 2 from Theorem 1, we use the methods of Wong's paper [9] and some known results about 2-groups.

Lemma 4. Suppose $S$ is a Sylow 2-subgroup of $G$ and $P<S$. Assume that $P$ is a Klein four-group and that $C_{S}(P)=P$. Then $S$ is a dihedral or a semi-dihedral groun.

Proof. Clearly, $Z(S)<P$, and $P=Z(S) \times<t>$ for some $t \in P$. Now $C_{S}(t)=C_{S}(P)=P$. By a result of Suzuki (Lemma 4, pp. 262-263 of [6]), $S$ must be dihedral or semi-dihedral.

Lemma 5. Suppose a Sylow 2-subgroup $S$ of $G$ has order 32 and $i$ generated by elements $x, y$, and $z$ satisfying

$$
x^{8}=y^{2}=z^{2}=[y, z]=1, \quad[x, y]=z, \quad[x, z]=x^{4} .
$$

Then $G$ has a normal 2-complement.

Proof. For every subgroup $H$ of $G$, let $H^{2}$ be the subgroup of $H$ generated by the elements $h^{2}, h \in H$. Since every group of exponent one or two is Abelian, $H / H^{2}$ is Abelian.

In page 244 of [9], it is proved that $G$ has a normal subgroup $G_{1}$ of index two such that 


$$
G_{1} \cap S=\left\langle x^{2}, y, z\right\rangle=\left\langle x^{2}, y\right\rangle \times<z>\cong D_{8} \times Z_{2} .
$$

Let $G_{2}=\left(G_{1}\right)^{2}$. By a result of Wielandt (Lemma $5(a)$ of [2]), $z \notin G_{2}$. Now, $G_{2}$ is characteristic in $G_{1}$ and therefore normal in $G$. Since $[x, y]=z, G / G_{2}$ is a non-Abelian 2-group. Thus $G / G_{2}$ has at least two generators, and

$$
\left|G / G^{2}=\right|\left(G / G_{2}\right) /\left(G / G_{2}\right)^{2} \mid \geqq 4 .
$$

However, $S^{2} \leqq S \cap G^{2}$ and $\left|S / S^{2}\right|=4$. Therefore,

$$
G^{2} \cap S=S^{2}=\left\langle x^{4}, z\right\rangle \cong Z_{4} \times Z_{2} \text {. }
$$

Hence $G^{2}$ has a Sylow 2-subgroup, $S^{2}$, that is contained in the center of its normalizer. By a result of Burnside ([4], p. 203), $G^{2}$ has a normal 2-complement. This complement must be a normal 2-complement for $G$.

Proof of Theorem 2. (I) Since $P$ is a Sylow 2-subgroup of $H$, $O_{2}(H) \subseteq P$. As $O_{2}\left(H / O_{2}(H)\right)=1,\left|H / O_{2}(H)\right|=2 m$ for some odd number $m$. Therefore, $H / O_{2}(H)$ is generated by two conjugates of $P / O_{2}(H)$. Take $a \in H$ such that $H=\left\langle P, P^{a}\right\rangle$. Then $P \cap P^{a}=O_{2}(H)$.

Let $\widetilde{G}=\left\langle H, H^{y}\right\rangle$, and let $Q$ be the largest normal subgroup of $\widetilde{G}$ contained in $P$. Take $b \in P$ and $c \in C(P)$ such that $y^{2}=b c$. Then $H^{y^{2}}=H^{b c}=H^{c}$. Therefore,

$$
H^{c}=H^{y^{2}} \leqq \widetilde{G}^{y} \leqq N\left(Q^{y}\right) \text {, and } H \leqq N\left(\left(Q^{y}\right)^{c-1}\right)=N\left(Q^{y}\right) \text {. }
$$

Hence $N\left(Q^{y}\right) \geqq<\widetilde{G}^{y}, H>\geqq<H^{y}, H>=\widetilde{G}$. Since $\left|Q^{y}\right|=|Q|, Q^{y}=Q$. By (I), $Q=1$. Thus $P \cap Z(\widetilde{G})=1$. In Corollary 1 we let $p=n=2$ and obtain $2 \leqq t \leqq 4$. The proof of Lemma 5 of [9] shows that $P$ is isomorphic to $Z_{2} \times Z_{2}, D_{8}$, or $D_{8} \times Z_{2}$.

Let $M$ be a cyclic subgroup of order $\frac{1}{2}\left|H / O_{2}(H)\right|$ in $H$, and let $N=O_{2}(H)$. Then $M N$ has index two in $H$ and $C_{M N}(N)=Z(N) \times C_{M}(N)$, so $K(H)=C_{M}(N)$. Thus $M / K(H)$ is isomorphic to a group of automorphisms of $N$. If $t=2$, then $|N|=2, M=K(H)$, and $H / K(H) \cong$ $P \cong Z_{2} \times Z_{2}$.

Suppose $t>2$. Then $P$ is non-Abelian and $P^{\prime} \leqq N$. Since $P$ and $y$ normalize $P^{\prime}$ and $H=P M, M$ does not normalize $P^{\prime}$. Therefore, $M / K(H) \neq 1$. If $t=3$, then $|N|=4$ and $|M / K(H)|=3$; therefore $|H / K(H) N|=6$ and $H / K(H) \cong S_{4}$. Suppose $t=4$. Then $|N|=8$ and the automorphism group of $N$ is not a 2-group. Since $D_{8} \times C_{2}$ has no quaternion subgroups, $N$ must be an elementary Abelian group. The automorphism group of $N$ contains a dihedral group of order $2 m^{\prime}$, with $m^{\prime}$ odd, only if $m^{\prime}=3$. Hence $|H| K(H) N \mid=6$. The proof of Lemma 6 of [9] shows that $H / K(H) \cong S_{4} \times Z_{2}$. 
(II) Let $T=N_{S}(P)$. Then $P<T$. If $t=2$, then $P=N_{S}(N)=$ $C_{S}(N)$, and therefore $P=C_{S}(P)$. If $t=3$, then $N \cong Z_{2} \times Z_{2}$ and $C_{S}(N)=N_{S}(N) \cap C_{S}(N)=C_{P}(N)=N$. By Lemma $4, S$ is a dihedral or semi-dihedral group in each of these cases.

Suppose $t=4$. From (I), $H$ does not have a normal 2-complement; hence, neither does $G$. Furthermore, $P \cong D_{8} \times Z_{2}$ and $N \cong Z_{2} \times Z_{2} \times Z_{2}$. It follows that $N$ has only two images under the automorphism group of $P$. Since $N_{S}(N)=P,|T / P|=2$. Thus $T=\langle P, y\rangle$. If $U \leqq S$ and $P<U$, then $P<N_{U}(P)$ and consequently $y \in T \leqq U$. Therefore, by hypothesis, $N_{S}\left(P_{0}\right)=P$ whenever $1<P_{0}<P$ and $P_{0}$ is a normal subgroup of $H$. Now by Lemma 8 of [9] and by Lemma $5, S$ has the desired form.

(III) In this case, there exists $y \in N_{S}(P)-P$ such that $y^{2} \in P$, so the results of (I) and (II) may be applied. This completes the proof of Theorem 2.

CoRollary 2. Assume the hypothesis of part (II) of Theorem 2. If $t=2$ or $t=3$, then $G$ satisfies one of the following conditions:

(i) $G$ has a normal 2-complement.

(ii) $G$ has a normal subgroup $G_{0}$ of index two, and $G_{0}$ has no normal subgroup of index two and has a dihedral Sylow 2-subgroup.

(iii) $G$ has a normal subgroup $G_{0}$ of index two, and $G_{0}$ has no normal subgroup of index two and has a generalized quaternion Sylow 2-subgroup. (In this case, $S$ must be a semi-dihedral group.)

(iv) $G$ has no normal subgroup of index two, and the elements of order two in $G$ are all conjugate in $G$.

If $t=2$ and $G$ satisfies (i) or (iii), then $C_{S}(K(G))=1$. If $t=3$, then $G$ cannot satisfy (i) or (iii). If $t=4, G$ satisfies one of the following conditions:

(v) $G$ has a normal subgroup $G_{1}$ of index two, and $G_{1}$ has no normal subgroup of index two and has a semi-dihedral Sylow 2subgroup.

(vi) $G$ has a normal subgroup $G_{2}$ of index four, and $G_{2}$ has no normal subgroup of index two and has a dihedral Sylow 2-subgroup.

Proof. If $t=2$ or $t=3$, then $S$ is dihedral or semi-dihedral, by Theorem 2. A dihedral group has no generalized quaternion subgroups. Therefore, $G$ satisfies one of the conditions (i) through (iv), by Lemma 8 of [3] (for $S$ dihedral) and by Lemma 1 and Theorem 2 of [8] (for $S$ semi-dihedral).

Let $K=K(G)$. Suppose $t=2, G$ satisfies (i) or (iii), and $C_{S}(K) \neq 1$. Then $P=N \times Z(S)$ and $Z(S) \$ Z(H)$. Since $|Z(S)|=2$ and $C_{S}(K)$ is normal in $S, Z(S) \subseteq C_{S}(K)$. However, $Z(S) K / K \leqq Z(G / K)$. (This requires the Brauer-Suzuki Theorem [1] if (iii) holds.) Therefore, 
$Z(S) K=Z(S) \times K$, so $Z(S)$ is the unique Sylow 2-subgroup of $Z(S) K$. As $Z(S) K$ is normal in $G, Z(S)$ is normal in $G$. Since $|Z(S)|=2$, $Z(S) \leqq Z(G)$. This is impossible, since $Z(S) \not \measuredangle Z(H)$.

Suppose $t=3$. Since $H$ does not have a normal 2-complement, (i) is impossible. Since $H^{\prime}$ contains the four-group $N$, (iii) is impossible.

Suppose $t=4$. The structure of $S$ is given in Theorem 2. The argument for Case ( $V$ ) of ([9], pp. 244-245) proves that $G$ has a normal subgroup $G_{1}$ of index two for which $S \cap G_{1}$ is semi-dihedral. Now the structure of $G_{1}$ is given by one of the conditions (i) through (iv). Since $H$ does not have a normal 2-complement, neither does $G_{1}$. Suppose $G_{1}$ has a normal subgroup $G_{2}$ of index two. Then $G_{2}$ is unique and $G_{2} \cap S$ is a dihedral or a quaternion group. Therefore, $G_{2}$ is normal in $G$. Since $\left|G / G_{2}\right|=4, G^{\prime} \leqq G_{2}$. As $H^{\prime} \cap N$ is a four-group, $G_{2} \cap S$ is not a quaternion group. So $G_{2} \cap S$ is a dihedral group of order eight. Now $G_{2} \cap S \leqq N_{S}\left(H^{\prime} \cap N\right)=P$. This completes the proof of Corollary 2.

We thank the Sloan Foundation for its support during the preparation of this paper. We also thank Professor I.M. Isaacs for suggesting the present form of Theorem 1 .

\section{REFERENCES}

1. R. Brauer, Some applications of the theory of blocks of characters of finite groups, II, J. of Algebra 1 (1964), 307-334.

2. G. Glauberman and J. G. Thompson, Weakly closed direct factors of Sylow subgroups, Pacific J. Math. 26 (1968), 73-83.

3. D. Gorenstein and J. Walter, On finite groups with dihedral Sylow 2-subgroups, Illinois J. Math. 6 (1962), 553-593.

4. M. Hall, The Theory of groups, Macmillan, New York, 1959.

5. C. C. Sims, Graphs and finite permutation groups, Math. Z. 95 (1967), 76-86.

6. M. Suzuki, A characterization of the simple groups $L F(2, p)$, J. Fac. Sci. Univ. Tokyo 6 (1951), 259-293.

7. H. Wielandt, Finite permutation groups, Academic Press, New York, 1964.

8. W. J. Wong, On finite groups whose 2-Sylow subgroups have cyclic subgroups of index 2, J. Austral. Math. Soc. 4 (1964), 90-112.

9. - Determination of a class of primitive permutation groups, Math. Z. 99 (1967), 235-246.

Received May 13, 1968.

UNiversity of Chicago

Chicago, Illinois 



\section{Pacific Journal of Mathematics \\ Vol. 29, No. 1 \\ May, 1969}

Jorge Alvarez de Araya, A Radon-Nikodým theorem for vector and operator

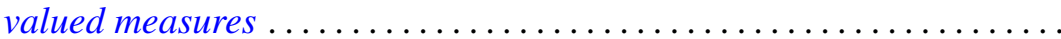

Deane Eugene Arganbright, The power-commutator structure of finite

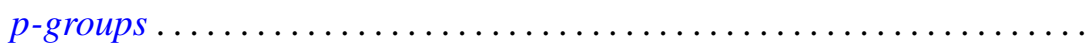

Richard Eugene Barlow, Albert W. Marshall and Frank Proschan, Some

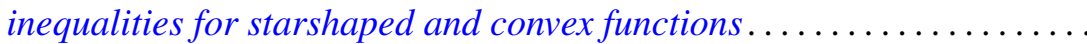

David Clarence Barnes, Some isoperimetric inequalities for the eigenvalues

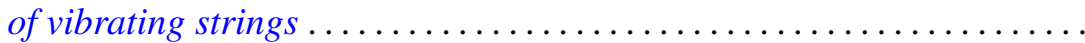

David Hilding Carlson, Critical points on rim-compact spaces ...........

Allan Matlock Weber Carstens, The lattice of pretopologies on an arbitrary

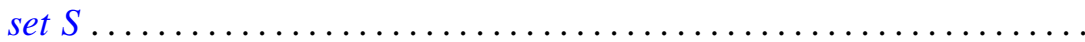

S. K. Chatterjea, A bilateral generating function for the ultraspherical

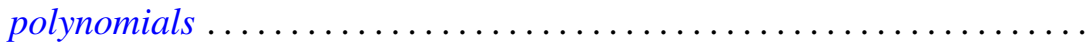

Ronald J. Ensey, Primary Abelian groups modulo finite groups ......... 77

Harley M. Flanders, Relations on minimal hypersurfaces ............ 83

Allen Roy Freedman, On asymptotic density in n-dimensions........... 95

Kent Ralph Fuller, On indecomposable injectives over artinian rings...... 115

George Isaac Glauberman, Normalizers of p-subgroups in finite groups . . . 137

William James Heinzer, On Krull overrings of an affine ring ........... 145

John McCormick Irwin and Takashi Ito, A quasi-decomposable abelian group without proper isomorphic quotient groups and proper isomorphic subgroups.

Allan Morton Krall, Boundary value problems with interior point boundary conditions

John S. Lowndes, Triple series equations involving Laguerre

polynomials

Philip Olin, Indefinability in the arithmetic isolic integers

Ki-Choul Oum, Bounds for the number of deficient values of entire functions whose zeros have angular densities..

R. D. Schafer, Standard algebras ....................

Wolfgang M. Schmidt, Irregularities of distribution. III.

Richard Alfred Tapia, An application of a Newton-like method to the Euler-Lagrange equation 